Results Of 10031 MSM attending MSHC, 58\% (95\% CI 57.4\%$59.4 \%)(5655 / 9677)$ had hepatitis B surface antibody (HepBsAb), $11 \%$ (95\% CI 10.0\%-11.4\%) (840/7888) had core antibody (HepBcAb) and 4\% (95\% CI 3.0\%-4.5\%) (95/2577) had surface antigen (HepBsAg). The proportion decreased, with HepBsAb from $72 \%$ to $48 \%[\mathrm{P}$ (trend) < 0.001], with HepBcAb from 12\% - 8\% $[\mathrm{P}$ (trend) $=0.039]$ and with either HepBsAb or HepBcAb, from $67 \%-50 \%$ [P (trend) < 0.001] from 2002 to 2012 but no change for HepBsAg $[\mathrm{P}$ (trend) $=0.08]$. Later year $(2007-12)$ of being tested (adjusted odds ratio (AOR) $0.65,95 \%$ confidence intervals(CI) $0.58-0.73$ ), more partners in last 12 months (AOR $1.19,95 \% \mathrm{CI}$ 1.06-1.34) and previous HIV test being performed (AOR 1.63, 95\% CI 1.43-1.81) were associated with immunity to hepatitis $B$. There incidence rate of hepatitis B (7 cases in 3540 per years of observation) in MSM over the period was 1.98 (95\% CI $0.79-4.07)$ per 1,000 person years.

Conclusion The data suggest that the current level of immunity of about $50 \%$ has been sufficient to prevent any significant hepatitis $B$ infection in the last 10 years. Maintaining adequate vaccination levels ( $\geq 50 \%$ ) in MSM is important if outbreaks are to be prevented.

\section{P3.123 HEPATITIS C TESTING AND INCIDENCE IN HIV-POSITIVE MEN WHO HAVE SEX WITH MEN IN MELBOURNE, VICTORIA. A RETROSPECTIVE COHORT STUDY}

\section{doi:10.1136/sextrans-2013-051184.0582}

\begin{abstract}
${ }^{1,2}$ M S C Lim, 1,2M Hellard, ${ }^{1} \mathrm{C}$ El-Hayek, ${ }^{1,3} \mathrm{M}$ Cuevas, ${ }^{4,3} \mathrm{C}$ Fairley, ${ }^{5} \mathrm{D}$ Leslie, ${ }^{6} \mathrm{~N}$ Roth, ${ }^{7} \mathrm{~B}$ Tee, ${ }^{1,2} \mathrm{M}$ Stoove. 'Burnet Institute, Melbourne, Australia; ${ }^{2}$ Monash University,

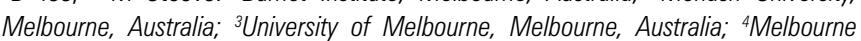
Sexual Health Centre, Melbourne, Australia; ${ }^{5}$ Victorian Infectious Diseases Laboratory,

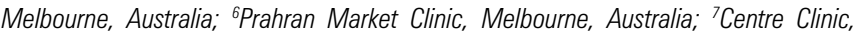
Victorian AIDS Council, Melbourne, Australia
\end{abstract}

Background Over the past five years there have been increasing reports of HCV transmission in HIV-positive men who have sex with men (MSM) globally. It is unclear whether this increase is due to increased transmission or increased detection (due to increased testing). This paper investigated reasons for increased HCV notification in HIV-positive MSM.

Methods HIV viral load test records between April 2006 and December 2011 were used to identify all HIV-positive patients attending three high MSM caseload clinics in Melbourne, Victoria. Their HCV test records were retrospectively linked over the same period. The following were determined: frequency of HCV testing; proportion of HIV-positive men tested for HCV annually; and HCV prevalence and incidence rate (per 100PY). Poisson regression calculated trends over time.

Results 3007 HIV-positive men attended the clinics; 2190 (73\%) were tested for HCV at least once, with 250 (11.4\%) testing HCV positive over the study period. The prevalence of co-infection declined significantly from $11.9 \%$ in 2006 to $7.4 \%$ in 2011 ( $p=0.01$ ). The number of HIV-positive men tested for HCV increased each year although the proportion tested remained the same (approximately $75 \%$ ) and testing frequency did not change (average 1.4 test/ person/year). 187 HIV-positive men were identified with HCV on their initial test and 63 incident infections were observed. HCV incidence among HIV-positive men was 1.55/100PY with no significant change over the study period.

Conclusions We found that HCV incidence in HIV-positive MSM remained stable and that prevalence decreased in HIV-positive MSM throughout the study period. Our results suggest the increase in HCV case notifications among HIV-positive men may be explained by an overall increase in HCV testing in this population. The steady increase in the number of HIV-positive MSM who remain well and consequently routinely tested for HCV may be contributing the increase in HCV notifications.

\section{P3.124 RISK FACTORS FOR HIV INFECTION AMONG MEN WHO HAVE SEX WITH MEN IN SEVEN CITIES IN COLOMBIA USING RESPONDENT-DRIVEN SAMPLING}

doi:10.1136/sextrans-2013-051184.0583

1 J Jacobson, ${ }^{2} \mathrm{~S}$ Morales Miranda, ${ }^{3} \mathrm{C}$ Sierra, ${ }^{4} \mathrm{R}$ Luque Núnez, ${ }^{3} \mathrm{M}$ Rubio Mendoza ${ }^{1}$ Independent consultant, Bogota, Colombia; 'Universidad del Valle de Guatemala, Guatemala City, Guatemala; 'United Nations Population Fund, Bogota, Colombia; ${ }^{4}$ Ministry of Health and Social Protection, Bogota, Colombia

Background Men who have sex with men (MSM) are increasingly recognised as the principal core group for HIV transmission in Latin America. In Colombia, factors associated with HIV infection have not previously been assessed.

Methods A secondary analysis was conducted using data from a bio-behavioural surveillance study among 2567 MSM ages 18 and older, recruited using respondent-driven sampling from seven cities in Colombia in 2010 ( $\mathrm{N}=333$ to 488 across cities). The study used a face-to-face survey and biological testing to assess risk behaviours and HIV infection. Factors with a bivariate association with infection were estimated from the pooled sample using a multilevel logistic regression framework, including random effects for recruitment tree and immediate recruiter, fixed effects to control for unobserved differences across cities, recruiter-level controls to account for recruitment patterns, and probability weights to account for differential personal and city-level estimated network size.

Results Estimated HIV prevalence ranged from 5.8\% in Pereira (95\% confidence interval [CI] $2.7 \%-9.5 \%$ ) to $23.7 \%$ in Cali (CI $17.6 \%-29.9 \%)$. Recruiter's HIV infection was moderately related to participant's HIV infection ( $\mathrm{OR}=1.5, \mathrm{CI}=0.9-2.5, \mathrm{P}=0.091)$. Clustering by recruiter, recruitment tree, and city explained $10 \%, 10 \%$ and $2 \%$ of variance in the HIV outcome, respectively. Factors associated with HIV $(\mathrm{P} \leq 5 \%)$ included age $\geq 40(\mathrm{OR}=2.9)$ and $25-39$ years $(\mathrm{OR}=2.5)$ compared to $18-24$ years, meeting casual sex partners at saunas $(\mathrm{OR}=2.6)$, previous sexually transmitted infection $(\mathrm{STI})(\mathrm{OR}=2.1)$, current employment $(\mathrm{OR}=1.9)$ and age of sexual debut $<18$ years $(\mathrm{OR}=1.4)$.

Conclusion The distribution of HIV infection among MSM in Colombia's largest cities suggests an ageing epidemic. HIV testing and prevention interventions should be intensified, target MSM at highest risk as well as younger age groups and STI infection to stem transmission early. Future analysis of this sample should also control for the hierarchical sampling structure and recruitment patterns.

\section{P3.125 INCREASING HIV INFECTION AMONG MEN WHO HAVE SEX WITH MEN IN SLOVENIA: SURVEILLANCE DATA FOR 2002-2011}

doi:10.1136/sextrans-2013-051184.0584

T Kustec, Z Kastelic, I Klavs. National Institute of Public Health, Ljubljana, Slovenia

Background HIV surveillance in Slovenia is based on universal mandatory reporting of HIV/AIDS cases, monitoring HIV infection prevalence among several sentinel populations and behaviour surveillance in several sentinel populations. Our objective was to present HIV surveillance data for men who have sex with men (MSM) in order to inform HIV prevention and control policies.

Methods We collected information on annual reported HIV cases, CD4 counts at diagnosis, HIV prevalence among male clients of STI outpatient services tested for syphilis and in a sentinel population of MSM, as well as proportion reporting "condom use" and "HIV testing last year" in the same sentinel population of MSM.

Results In 2011, 35 of all 55 newly diagnosed HIV cases were reported among MSM. During 2002-2011, the annual reported incidence rate of HIV diagnoses among MSM increased from 12.6 to 48.0 per million men aged 15-64 years. The proportion of new HIV diagnosis among MSM that were late $\left(\mathrm{CD} 4\right.$ counts $\left.<350 / \mathrm{mm}^{3}\right)$ varied between the 